\title{
Microstructure and Electrochemical Performance of 3D Porous Graphene
}

\author{
HOU Zhao Xia ${ }^{\text {a }}$, SUN Dan, LI Lin, WANG Mei Han, HU Xiao Dan and LONG \\ Hai Bo
}

Liaoning Province Key Laboratory of New Functional Materials and Chemical Technology, School of Mechanical Engineering, Shenyang University, Shenyang 110044, China

a luckyxia2007@126.com

Keywords: Graphene; Microstructure; Electrochemical Performance; Porous.

Abstract. 3D porous graphene was fabricated by hydrothermal cross-linking method and template method, respectively. X-ray diffraction, scanning electron microscopy, fourier transform infrared spectrometry, BET, cyclic voltammetry and electrochemical impedance spectroscopy are used to characterize the microstructure and electrochemical performance. The pore size is mainly in mesoporous range. The specific surface area and the pore volume of porous 3DrGO-hydrothermal is better than that of porous $3 \mathrm{DrGO}-\mathrm{CaCO}_{3}$, the pore size is in a narrow range and more uniform. The specific capacitance of $3 \mathrm{DrGO}$ hydrothermal is better than $3 \mathrm{DrGO}-\mathrm{CaCO}_{3}$.

\section{Introduction}

Carbon as a key element of nature, first appeared in "Periodic table" edited by Walaxi in 1789 [1]. In 1985, scientists in the United States, Kroto, found that the zero-dimensional fullerene C60 [2]. In 1991, Lijima team firstly found that the one-dimensional carbon nanotubes [3]. Since 2004, Andre Geim and Konstantin Novoselov, physicists from the University of Manchester in the United Kingdom, firstly separated the two-dimensional crystal structure from graphite, which was known as "graphene", the most thinnest and hardest materials so far, and won 2010 Nobel prize in Physics [4].

Graphene, as two-dimensional (2D) carbon material, its structure is a monolayer of carbon atoms closely packed together to form the 2D honeycomb crystal. Because of the exceptional physical and chemical properties of $2 \mathrm{D}$ graphene, it has great practical value in the field of information transmission, resource utilization, material recovery and medical biology, etc. However, graphene materials are also expected to get better development and application if it can be assembled to 3D porous structure. From the micro perspective, in additon to the inherent properties of two dimensional graphene, 3D porous material has many excellent properties, such as large surface area, fast transmission, high load and activity of the catalyst, which have fully extended application field [5].

In this paper, it focuses on the preparation and characterization of $3 \mathrm{D}$ porous graphene by hydrothermal and template methods.

\section{Experimental}

Materials. The experimental materials are all analytical purity and used as received without further purification.

Synthesis. Graphene oxide (GO) were prepared by modified hummer's method. 3D porous reduction graphene oxide (3DrGO) were prepared by hydrothermal crosslinking and $\mathrm{CaCO}_{3}$ template method, respectively. The samples were marked by 3DrGO-hydrothermal and $3 \mathrm{DrGO}-\mathrm{CaCO}_{3}$, respectively. 3DrGO-hydrothermal were synthesized by hydrothermal treatment of $\mathrm{GO}$ at $180^{\circ} \mathrm{C}$ for $24 \mathrm{~h}$. Self-assembled graphene hydrogel was shown in Fig. 1 (a). A large amount of water was used to rinse again and again, and finally dried for $48 \mathrm{~h}$ by the vacuum freeze. $3 \mathrm{DrGO}-\mathrm{CaCO}_{3}$ was prepared by using glucose as the reducing agent and $\mathrm{CaCO}_{3}$ as the template. Firstly, the mixture of $\mathrm{Na}_{2} \mathrm{CO}_{3}$ and ethanol was slowly dropped into $\mathrm{CaCl}_{2}$ with stirring to form part A. Glucose and ammonium hydroxide were added into GO solution to get part B. And then, the mixture of A and B was poured 
into teflon-lined autoclave and heattreated at $100{ }^{\circ} \mathrm{C}$ for $40 \mathrm{~min}$. Subsequently, heated to $180{ }^{\circ} \mathrm{C}$ for $24 \mathrm{~h}$. The hydrogel was shown in Fig. 1 (b). Then the hydrogel was placed into $1 \mathrm{M} \mathrm{HCl}$ solution for 1 $\mathrm{h}$, followed by filtering, washing and vacuum freeze drying to obtained $3 \mathrm{DrGO}-\mathrm{CaCO}_{3}$.
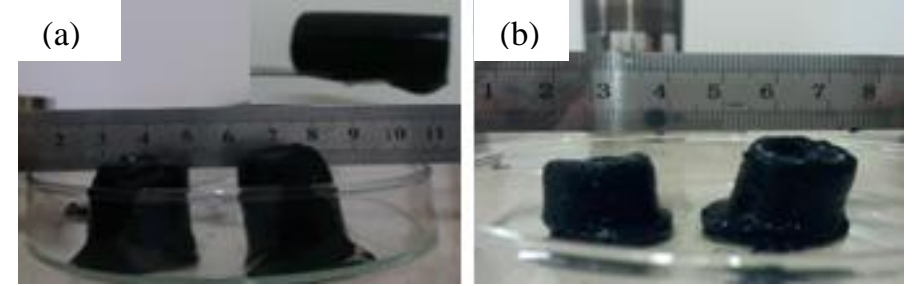

Fig. 1 The graphene hydrogel by hydrothermal crosslinking method (a) and template method (b)

Characterization. X-ray diffraction (XRD) was recorded using Holland BV PW 3040/60 diffractometer with $\mathrm{Cu} \mathrm{K \alpha}$ radiation $(\lambda=0.154 \mathrm{~nm})$ at $40 \mathrm{kV}$ and $40 \mathrm{~mA}$ with a scanning rate of $5 \% \mathrm{~min}$ from $5^{\circ}$ to $60^{\circ}$. Foutier transform infrared spectrometer (FT-IR) was recorded with Bruker IFS-55 FT-IR spectrometer at room temperature from $400 \mathrm{~cm}^{-1}$ to $4000 \mathrm{~cm}^{-1}$. The morphology of as-prepared samples was characterized by a scanning electron microscope (SEM) of Japan S-4800. The $\mathrm{N}_{2}$ adsorption and desorption isotherms were recorded at $77 \mathrm{~K}$ with a Micromeritics ASAP 2020 (USA). Specific surface area was calculated by a Braunauer-Emmett-Teller (BET) method and the pore size distribution was determined from the adsorption branches of the isotherms using the Barrett-Johner-Halendar (BJH) theories. All the electrochemical performance was studied on a PARSTART 2237 electrochemical workstation(China). Cyclic voltammetry (CV) and electrochemical impedance spectroscopy (EIS ) measurments were carried out in a two-electrod system with $1 \mathrm{M} \mathrm{H}_{2} \mathrm{SO}_{4}$ aqueous electrolyte, a cellulose paper as separator and carbon fibre paper as current collector.

\section{Results and discussion}

Morphology. The morphology of 3DrGO-hydrothermal and $3 \mathrm{DrGO}-\mathrm{CaCO}_{3}$ are characterized by SEM, as shown in Fig. 2. 3DrGO-hydrothermal is shown in Fig.2 (a) and (b). The surface morphology of the 3DrGO-hydrothermal sample shows the porous structure after hydrothermal treatment.The pore walls consist of thin layers of stacked graphenen sheets. The pore sizes are at the range of a few nanometers to tens of nanometers. The partial overlapping or coalescing of flexible graphene sheets results in the formation of physical cross-linking sites of the framework. Under hydrothermal environment, internal oxygen containing functional groups are removed, and the electrostatic force and the hydrophilic force are decreased, the interaction between the sheets is close to each other, and the cross linking action makes them self-assembled into more stable 3D structure.

3DrGO-CaCO${ }_{3}$ SEM photographs are shown in Fig. 2 (c) and (d). It can be seen that rGO remained the 3D porous morphology after removal of $\mathrm{CaCO}_{3}$ template. Edge and layers stack can be observed, which is due to the $\pi-\pi$ acting force between rGO sheets. The pore size and its distribution are bigger than that of rGO-hydrothermal.
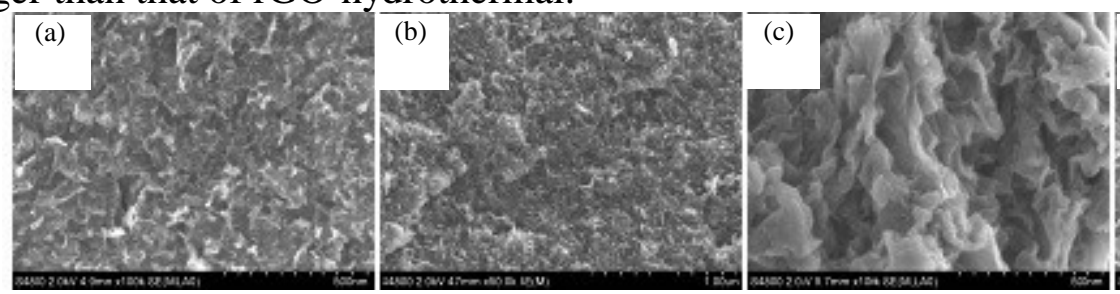

(d)

Fig. 2 SEM photographs of 3DrGO-hydrothermal (a, b) and 3DrGO-CaCO 3 (c, d)

Phase analysis. XRD patterns of 3DrGO-hydrothermal and $3 \mathrm{DrGO}-\mathrm{CaCO}_{3}$ are shown in Fig 3. No $\mathrm{CaCO}_{3}$ diffraction peak can be observed for $3 \mathrm{DrGO}-\mathrm{CaCO}_{3} \mathrm{XRD}$ curve, which indicates that $\mathrm{CaCO}_{3}$ template are etched completely. The XRD patterns are similar, just little difference for $2 \theta$, $3 \mathrm{DrGO}$-hydrothermal is $25.09^{\circ}$, while $3 \mathrm{DrGO}-\mathrm{CaCO}_{3}$ is $25.24^{\circ}$. The interlayer spacings are 0.3546 $\mathrm{nm}$ and $0.3526 \mathrm{~nm}$, respectively. The diffraction peaks of 3DrGO-hydrothermal are higher and wider 
than that of 3DrGO-CaCO , which indicates that 3DrGO-hydrothermal stacked more randomly and distributed more disorderly. The difference can be attributed to the different reduction method.

FT-IR spectra Analysis. The FT-IR spectra of 3DrGO-hydrothermal and $3 \mathrm{DrGO}-\mathrm{CaCO}_{3}$ are shown in Figure 4. 3DrGO-hydrothermal and $3 \mathrm{DrGO}-\mathrm{CaCO}_{3}$ have similar FT-IR spectra. After reduction, the O-H peak at $3500-3000 \mathrm{~cm}^{-1}$ is still observable, and $2960-2850 \mathrm{~cm}^{-1}$ for $\mathrm{C}-\mathrm{H}$ stretching can be observed in both samples. while the carbonyl peak at $1718 \mathrm{~cm}^{-1}$ is inconspicuous for 3DrGO-hydrothermal samples, which indicates that the reduction effect of glucose to carbonyl is inferior to that of hydrothermal method.
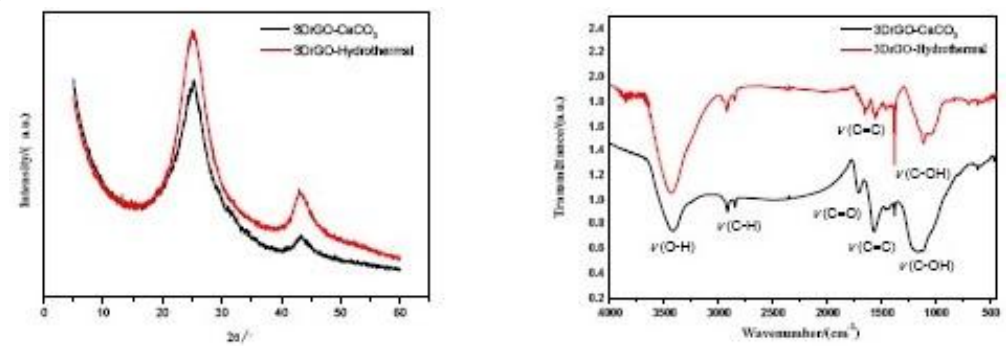

Fig. 3 XRD patterns of 3DrGO-hydrothermal and 3DrGO-CaCO 3 (Left)

Fig. 4 FT-IR spectra of 3DrGO-hydrothermal and 3DrGO-CaCO 3 (Right)

Specific surface area and pore analysis. The nitrogen adsorption/desorption isotherms and pore size distribution for $3 \mathrm{DrGO}$ hydrothermal $(\mathrm{a}, \mathrm{b})$ and $3 \mathrm{DrGO}-\mathrm{CaCO}_{3}(\mathrm{c}, \mathrm{d})$ were shown in Fig. 5 and table 1. It can be seen from Fig. 5 (a) that 3DrGO-hydrothermal exhibited irreversible type IV adsorption-desorption isotherms, and $\mathrm{H} 2$ hysteresis loop indicates that samples are porous structure. In the relative pressure $\mathrm{P} / \mathrm{P}_{0}=0.0-0.6$ low voltage and medium voltage area, absorption and desorption curves rise rapidly, indicates that samples are microporous and mesoporous structure. In the relative pressure $\mathrm{P} / \mathrm{P}_{0}=0.4-0.7, \mathrm{H} 2$ hysteresis loop is associated with the filling of the mesopores due to capillary condensation.
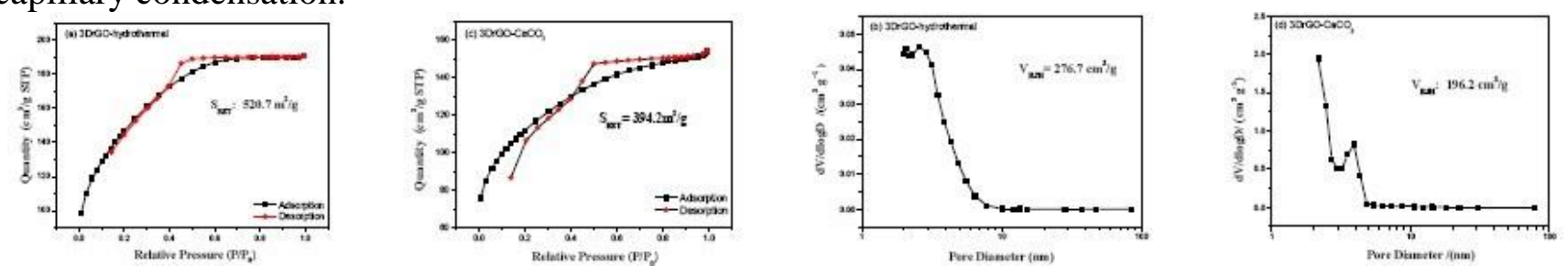

Fig. 5 The nitrogen adsorption/desorption isotherms and pore size distribution for

3DrGO-hydrothermal (a, b) and 3DrGO-CaCO $3(\mathrm{c}, \mathrm{d})$

It can be seen from Fig. 5(c) that $3 \mathrm{DrGO}-\mathrm{CaCO}_{3}$ exhibited irreversible type IV adsorptiondesorption isotherms, and $\mathrm{H} 2$ hysteresis loop indicates that samples are porous structure. In the relative pressure $\mathrm{P} / \mathrm{P}_{0}=0.0-0.1$ low voltage area, absorption and desorption curves are close to $\mathrm{Y}$ axis, which indicates that there exists strong force between this sample and nitrogen, it is due to the strong adsorption potential of micropores. In the relative pressure $\mathrm{P} / \mathrm{P}_{0}=0.4-0.9, \mathrm{H} 2$ hysteresis loop is associated with the filling of the mesopores due to capillary condensation.

Table 1 summarized the physical parameters that calculated from nitrogen adsorption-desorption isotherms. The surface area of $3 \mathrm{DrGO}$ hydrothermal is larger than that of $3 \mathrm{DrGO}-\mathrm{CaCO}_{3}$. Micropore volume of both samples are far less than total pore volume, which indicates that the pore size is mainly in mesoporous range.

Table 1 Textural properties of 3DrGO hydrothermal and 3DrGO-CaCO${ }_{3}$

\begin{tabular}{|c|c|c|c|c|}
\hline samples & $\mathrm{S}_{\mathrm{g}} /\left[\mathrm{m}^{2} \mathrm{~g}^{-1}\right]^{\mathrm{a}}$ & $\mathrm{V}_{\text {total }} /\left[\mathrm{cm}^{3} \mathrm{~g}^{-1}\right]^{\mathrm{b}}$ & $\mathrm{V}_{\mathrm{mic}} /\left[\mathrm{cm}^{3} \mathrm{~g}^{-1}\right]^{\mathrm{c}}$ & $\mathrm{D}_{\mathrm{BJH}} /[\mathrm{nm}]^{\mathrm{d}}$ \\
\hline 3DrGO-hydrothermal & 520.7 & 0.29 & 0.055 & 2.7 \\
\hline 3DrGO-CaCO 3 & 394.2 & 0.24 & 0.055 & 3.1 \\
\hline
\end{tabular}

${ }^{\mathrm{a}}$ BET method; ${ }^{\mathrm{b}} \mathrm{N}_{2}$ adsorption total pore volume at $\mathrm{p} / \mathrm{p}_{0}=0.975 \sim 0.976$

${ }^{\mathrm{c}}$ t-Plot micropore volume; ${ }^{\mathrm{d}} \mathrm{BJH}$ adsorption average pore diameter

Electrochemical performance. The specific capacitances (Cs), i.e. the capacitance per unit mass of the active materials in the electrodes, of the samples were calculated from both cyclic 
voltammetry(CV, Fig. 6) using a two-electrode set-up at different scan rates. The curves appear as quasi-rectangular at low scan rate, and started to tilt when the scan rate increased. The Cs of 3DrGO hydrothermal and $3 \mathrm{DrGO}-\mathrm{CaCO}_{3}$ are $118 \mathrm{~F} / \mathrm{g}$ and $37 \mathrm{~F} / \mathrm{g}$ at $5 \mathrm{mV} / \mathrm{s}$, respectively.

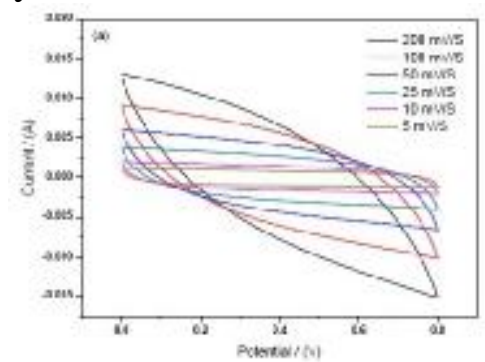

Fig. $6 \mathrm{CV}$ curves of 3DrGO hydrothermal (a) and

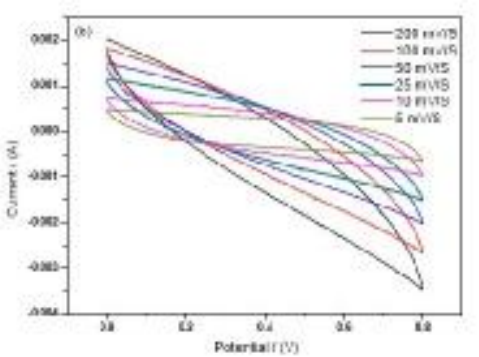

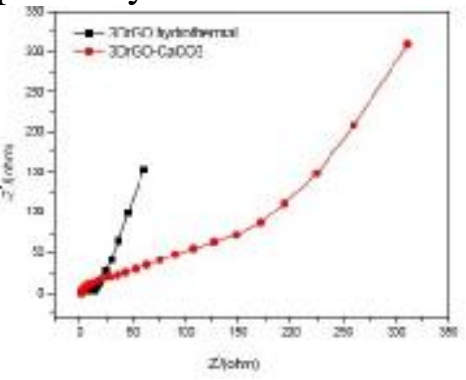

Fig. 7 Nyquist plot

The electrochemical properties of samples were further characterized by electrochemical impedance spectroscopy (EIS, Fig. 7). The low frequency regions of the EIS curve of 3DrGO hydrothermal appears more perpendicular towards the $\mathrm{Z}^{\prime}$ axis, indicating a more ideal capacitance behavior. The first and second intersects of the curves to the $\mathrm{Z}^{\prime}$ axis at high frequency regions of EIS curves represent equivalent series resistance $\left(R_{\mathrm{s}}\right)$ as well as the sum of Rs and charge transfer resistance (Rct), respectively. The value of the samples is listed in Table 2. The Rct of $3 \mathrm{DrGO}-\mathrm{CaCO}_{3}$ is bigger than that of 3DrGO hydrothermal, the more Rct lead to the low Cs for $3 \mathrm{DrGO}-\mathrm{CaCO}_{3}$. The more Ret may be due to the reduce result of glucose is inferior to that of hydrothermal method.

Table $2 R_{s}$ and $R_{c t}$ extracted from Nyquist plot in Fig.7.

\begin{tabular}{ccc}
\hline sample & 3DrGO hydrothermal & 3DrGO-CaCO \\
\hline $\mathrm{R}_{\mathrm{s}}(\mathrm{ohm})$ & 1.30 & 1.32 \\
$\mathrm{R}_{\mathrm{ct}}(\mathrm{ohm})$ & 8.60 & 55.2 \\
\hline
\end{tabular}

\section{Conclusions}

3D rGO were prepared by hydrothermal cross-linking and template methods. Hydrothermal crosslinking is at $180{ }^{\circ} \mathrm{C}$ for $24 \mathrm{~h}$. The template method used $\mathrm{CaCO}_{3}$ as the template and glucose as the reductant, and the template was etched by hydrochloric acid solution. Both methods could successfully assemble the $2 \mathrm{D}$ graphene into $3 \mathrm{D}$ porous structure. The specific surface area of $3 \mathrm{DrGO}-$ hydrothermal $\left(520.7 \mathrm{~m}^{2} \mathrm{~g}^{-1}\right)$ is larger than that of 3DrGO-CaCO $3\left(394.2 \mathrm{~m}^{2} \mathrm{~g}^{-1}\right)$. 3DrGO-hydrothermal and $3 \mathrm{DrGO}-\mathrm{CaCO}_{3}$ mainly dominated by mesopores. The specific capacitance of 3DrGO hydrothermal and $3 \mathrm{DrGO}-\mathrm{CaCO}_{3}$ are $118 \mathrm{~F} / \mathrm{g}$ and $37 \mathrm{~F} / \mathrm{g}$ at $5 \mathrm{mV} / \mathrm{s}$, respectively.

\section{Acknowledgements}

This work was financially supported by the Program of Natural Science Foundation of China (No. 51472166) and Liaoning BaiQianWan Talents program.

\section{References}

[1] J. Su, M. Cao, L. Ren, et al:ournal of Physical Chemistry C vol.115 (2011), p14469

[2] J. Balach, L. Tamborini, K. Sapag, et al: Colloids \& Surfaces A Physicochemical \& Engineering Aspects Vol.415(2012), p343

[3] J.J. Moore, H.K. Jin, J. Z. Wen: Materials Chemistry \& Physics Vol.134(2012), p68

[4] A.K. Geim, K.S. Novoselov: Nature Materials Vol 6(2007), p183

[5] S. Nardecchia, D. Carriazo, M.L. Ferrer, et al: Chemical Society Reviews Vol. 42(2012), p794 